\title{
Gastrointestinal stromal tumors in Japanese patients with neurofibromatosis type I
}

\author{
Toshirou Nishida ${ }^{1,3} \cdot$ Masahiko Tsujimoto $^{2} \cdot$ Tsuyoshi Takahashi $^{4} \cdot$ \\ Seiichi Hirota ${ }^{5}$ Jean-Yves Blay ${ }^{6}$ Mari Wataya-Kaneda ${ }^{7}$
}

Received: 15 July 2015/ Accepted: 6 October 2015/Published online: 29 October 2015

(c) The Author(s) 2015. This article is published with open access at Springerlink.com

\begin{abstract}
Background Neurofibromatosis type I (NF1) predisposes patients to various neoplasias, including gastrointestinal stromal tumors (GISTs). Little is known about the risk of developing GISTs for NF1 patients or the clinicopathologic features and prognosis of NF1-GIST.

Methods We conducted a multi-detector computed tomography screen for adult NF1 patients between 2003 and 2012. Clinicopathologic data of sporadic GISTs from patients who underwent surgery between 2001 and 2010 were retrospectively collected from 32 hospitals in Japan. Results CT screening identified 6 GIST patients from the 95 NF1 patients screened, suggesting that the prevalence rate of GISTs was approximately 6.3/100 in NF1 patients. All 6 NF1 patients exhibited hyperplasia of the interstitial
\end{abstract}

Electronic supplementary material The online version of this article (doi:10.1007/s00535-015-1132-6) contains supplementary material, which is available to authorized users.

\section{Toshirou Nishida}

tnishida@east.ncc.go.jp

1 Department of Surgery, Osaka Police Hospital, Osaka, Japan

2 Department of Pathology, Osaka Police Hospital, Osaka, Japan

3 Department of Surgery, National Cancer Center Hospital East, 6-5-1 Kashiwanoha, Kashiwa, Chiba 277-8577, Japan

4 Department of Surgery, Osaka University Graduate School of Medicine, Osaka, Japan

5 Department of Surgical Pathology, Hyogo College of Medicine, Nishinomiya, Hyogo, Japan

6 Centre Leon Bernard of the Université Claude Bernard, Lyon, France

7 Department of Dermatology, Osaka University Graduate School of Medicine, Osaka, Japan cells of Cajal in the adjoining small intestine. NF1-GISTs may account for 1.1-1.3\% of primary sporadic GISTs and present as multiple tumors in the small intestine, with low mitotic activity and no KIT or PDGFRA mutations. The risk of recurrence and mortality is very similar between NF1 and non-NF1 patients after surgical resection of GISTs.

Conclusions NF1 patients may be predisposed to developing small intestinal GISTs, which may appear as multiple GISTs without KIT and PDGFRA mutations. The prognosis of patients with NF1-GISTs is similar to patients with conventional GISTs.

Keywords Neurofibromatosis type I - Gastrointestinal stromal tumor · Prognosis - Clinicopathologic features

$\begin{array}{ll}\text { Abbreviations } \\ \text { GIST } & \text { Gastrointestinal stromal tumor } \\ \text { IHC } & \text { Immunohistochemistry } \\ \text { ICC } & \text { Interstitial cells of Cajal } \\ \text { MDCT } & \text { Multi-detector computed tomography } \\ \text { MPNST } & \text { Malignant peripheral nerve-sheath tumors } \\ \text { NF1 } & \text { Neurofibromatosis type I } \\ \text { NF1-GIST } & \text { GIST associated with NF1 } \\ \text { OS } & \text { Overall survival } \\ \text { PD } & \text { Progressive disease } \\ \text { RFS } & \text { Recurrence-free survival } \\ \text { SDH } & \text { Succinate dehydrogenase }\end{array}$

\section{Introduction}

Gastrointestinal stromal tumors (GISTs) predominantly occur in the stomach (60-70\%) and small intestine (20-30\%), and multiple tumors are rarely observed [1, 2]. 
GIST proliferation may be caused by gain-of-function mutations in either the KIT (80-85\%) or PDGFRA $(10 \%)$ genes [2]. Five to ten percent of GISTs lack mutations in these genes (wild-type GISTs). Such wild-type GISTs are now known to be heterogeneous and may actually possess mutations in the $S D H$ complex genes, NF1, BRAF, NRAS, or HRAS. Imatinib mesylate, a selective tyrosine kinase inhibitor of KIT, PDGFR- $\alpha$, and Abl, exhibits exceptional activity in advanced GIST patients. The benefits of imatinib have largely been observed in KIT- and PDGFRAmutant GISTs; however, its activities are less well documented in wild-type GISTs [2].

Neurofibromatosis type I (NF1), also known as von Recklinghausen disease, is an autosomal dominant inherited syndrome affecting 1/3000-4000 individuals [3]. NF1 is distinguished by a variety of characteristic features and is clinically diagnosed using the National Institutes of Health diagnostic criteria [4]. NF1 is believed to be caused by functionally biallelic losses of the tumor suppressor gene $N F 1$, resulting in the functional loss of neurofibromin. NF1 patients are predisposed to both benign and malignant tumors, including neurofibroma, optic-pathway glioma, malignant peripheral nerve-sheath tumors (MPNSTs), neuroblastoma, GIST, pheochromocytoma, and breast cancer [3].

A number of case reports and small case series have indicated a strong association between NF1 and GIST [3, 5].The clinicopathologic features of GISTs associated with NF1 are different from those of sporadic GISTs. Most NF1 patients, for instance, exhibit multiple GISTs in the small intestine, and NF1-GISTs rarely exhibit mutations in the $K I T$ and PDGFRA genes [3, 7]. However, the existing studies of NF1-GISTs have been primarily conducted in Caucasian populations and are mostly case series with small numbers of patients or case reports. Some conflicting results were observed from these reports, [9, 10] and the incidence of GISTs in Japanese NF1 patients and the clinicopathologic features of NF1-GISTs in a Japanese population have not yet been established. Furthermore, to date, no report has demonstrated the outcomes of surgery and prognosis for NF1-GIST patients.

Although NF1 may be associated with an increased risk for GIST, little is known about the lifetime risk of developing a GIST in NF1 patients. Moreover, the frequency of NF1-GIST development among sporadic GISTs and the clinicopathologic characteristics and prognosis of NF1GIST remain unknown. In this study, we, therefore, evaluated NF1 patients by CT screening, and retrospectively analyzed the clinical and pathological characteristics of any identified NF1-GISTs. We also assessed NF1-GIST patient prognosis.

\section{Patients and methods}

\section{NF1 patient screening with multi-detector CT (MDCT)}

Because of the suspected high incidence of intestinal GISTs in adult NF1 patients, we planned and conducted multi-detector computed tomography (MDCT) screening. We recommended MDCT to adult NF1 patients over 30 years old who visited the Department of Dermatology at the Osaka University Hospital as well as to NF1 patients with signs and symptoms of the disease. The MDCT screening was offered to 158 NF1 patients between April 2003 and September 2012. Of these 158 NF1 patients, informed consent was obtained from 95, and they received MDCT with or without contrast media. We recommended the use of contrast media; however, when patients had a suspected allergy to the media or refused its use, a plain CT was performed. The details of the scanning protocol were similar to those described elsewhere [16]. The diagnosis of NF1 was confirmed according to the NIH diagnostic criteria [4]. Data were prospectively collected and retrospectively analyzed. This study was approved by the institutional review board of Osaka University Hospital and was conducted according to institutional ethics guidelines.

\section{Retrospective cohort study of sporadic GISTs}

We retrospectively collected NF1-GISTs and sporadic GISTs from 29 community hospitals and 3 institutions in Japan between 2001 and 2010. We sent survey forms to 32 hospitals and institutes, which identified 23 primary NF1GIST patients among 1314 patients with a primary GIST who underwent surgery during this period.

Clinical and pathological data for these NF1-GISTs were collected with tissue samples for pathological examination when available. Clinicopathologic data for sporadic GISTs other than those in NF1-GIST patients were obtained from 19 hospitals. Detailed clinicopathologic data were obtained from 927 of the 1314 primary sporadic GIST patients. This study was approved by the institutional review board of Osaka University Hospital and Osaka Police Hospital and was conducted according to institutional guidelines. NF1-GISTs were also identified from a database of reference centers for sarcoma in France (NetSARC, netsarc.org), which has now gathered over 21,000 sarcoma tumors since 2010. This database, updated in real-time by the 26 reference centers in France, collects data on an estimated $85 \%$ of the sarcoma cases in this country. 


\section{Pathological diagnosis and mutational analysis}

Tissue samples fixed with $10 \%$ buffered formalin and embedded in paraffin were sectioned ( $3 \mu \mathrm{m}$ thick) and used for hematoxylin and eosin staining (H\&E) and immunohistochemistry (IHC). IHC was performed using the ENVISION + KIT HRP (DAB) system (DAKO, Glostrup, Denmark) as previously described [17]. Histopathologic features, including cell shape, mean mitotic number per 50 $\mathrm{HPF}$, and immunophenotype, were analyzed by H\&E and IHC.

When freshly frozen samples were available, total RNA was extracted using the RNeasy Mini Kit (QIAGEN, Valencia, CA, USA). All coding regions of the KIT and/or PDGFRA genes were amplified by polymerase chain reaction (PCR) after reverse transcription of the extracted RNA as previously described [17, 18]. When only paraffin blocks were available, genomic DNA was extracted from the paraffin sections using the QIAamp DNA Mini Kit (QIAGEN). Exons 9, 11, 13, and 17 of the KIT gene and exons 12,14, and 18 of the PDGFRA gene were amplified by PCR using previously described primers [17, 19].

\section{Statistical analysis}

Statistical analyses were performed using the Chi squared test, Fisher's exact test, Student's $t$ test, and the MannWhitney $U$ test. The recurrence-free survival (RFS) was calculated from the date of surgery to the date of first tumor recurrence or to the date of death, censoring living patients without recurrence at the time of data collection. The overall survival (OS) was calculated from the date of surgery to the date of death, censoring living patients. RFS and OS between the groups were compared using the Kaplan-Meier life-table method with the log-rank test. The $p$ values were two-sided, and $p$ values $<0.05$ were considered significant. The data were analyzed using the Statistical Package for Social Sciences, version 17.0 (SPSS Inc., Chicago, IL, USA).

\section{Results}

\section{MDCT screening of NF1 patients}

Ninety-five NF1 patients, comprising 35 males and 60 females with a median age of 45 years (range 17-80), received MDCT with or without contrast media. One patient (a 66-year-old male) exhibited anemia in a prescreening blood examination, but all other patients did not exhibit gastrointestinal symptoms or signs before screening. Radiographic findings of neoplastic lesions included 6 gastrointestinal tumors (6.3\%; CI: 2.35-13.2\%), which were later diagnosed as GISTs after pathological examination of surgical specimens, 6 myoma uteri, 3 adrenal tumors, 3 intrapelvic masses suspected to be neurofibromas, and 1 mediastinal tumor (Table 1).

All 6 NF1 patients with gastrointestinal tumors underwent surgery. At the time of surgery, 5 of the 6 NF1 patients had multiple GISTs in the duodenum, jejunum, and/or ileum, and 1 patient had a single tumor in the jejunum (Fig. 1a; Table 2). Pathologically, all resected tumors were KIT-positive, DOG1-positive spindle cell tumors with few mitotic figures. Furthermore, pathological examination revealed that all 6 NF1 patients exhibited hyperplasia of the interstitial cells of Cajal (ICC) in the normal-appearing adjoining small intestine (Fig. 1b). KIT and PDGFRA genetic analysis revealed that no resected tumors exhibited mutations in either gene (Table 2).

\section{NF1-GISTs represent a rare sub-fraction of sporadic GISTs}

Next, we collected histologically confirmed GISTs, including NF1-GISTs, from patients who underwent surgery between 2001 and 2010 at 32 hospitals in Japan. In total, 1314 primary GISTs were collected, including 23 primary NF1-GISTs (1.75\%; CI: $1.17-2.61 \%)$. Six of the 23 NF1-GISTs were detected by CT screening, and when these 6 NF1-patients were excluded from the analysis, NF1-GISTs accounted for $1.30 \%$ (CI: 0.81-2.07\%) of the sporadic GISTs. A similar investigation was performed using data obtained from the French Netsarc/Rreps networks study. In this registry, 18 NF1-GIST patients $(1.1 \%$; CI: $0.75-1.85 \%$ ) were identified among 1528 GIST cases. Thus, NF1-GISTs are estimated at $1.1-1.3 \%$ of primary sporadic GISTs.

Clinicopathologic features were compared between the non-NF1-GISTs and NF1-GISTs (Table 3). The NF1GISTs more frequently presented as multiple lesions preferentially located in the small intestine compared with those of the non-NF1-GISTs. R0 or R1 surgery was less frequently performed for NF1-GIST patients than for nonNF1-GIST patients. The mitotic activity of the NF1-GISTs

Table 1 Abdominal lesions indicated by MDCT screening

\begin{tabular}{ll}
\hline Lesions & $N(\%)$ \\
\hline GIST & $6(6.3 \% ;$ CI: $2.35-13.24)$ \\
Myoma uteri & $6(6.3 \%, 10 \%$ for female $)$ \\
Adrenal tumor & $3(3.2 \%)$ \\
Suspected pelvic neurofibroma & $3(3.2 \%)$ \\
Mediastinal tumor & $1(1.0 \%)$ \\
Gallstone & $4(4.2 \%)$ \\
Renal artery aneurysm & $1(1.0 \%)$ \\
\hline
\end{tabular}




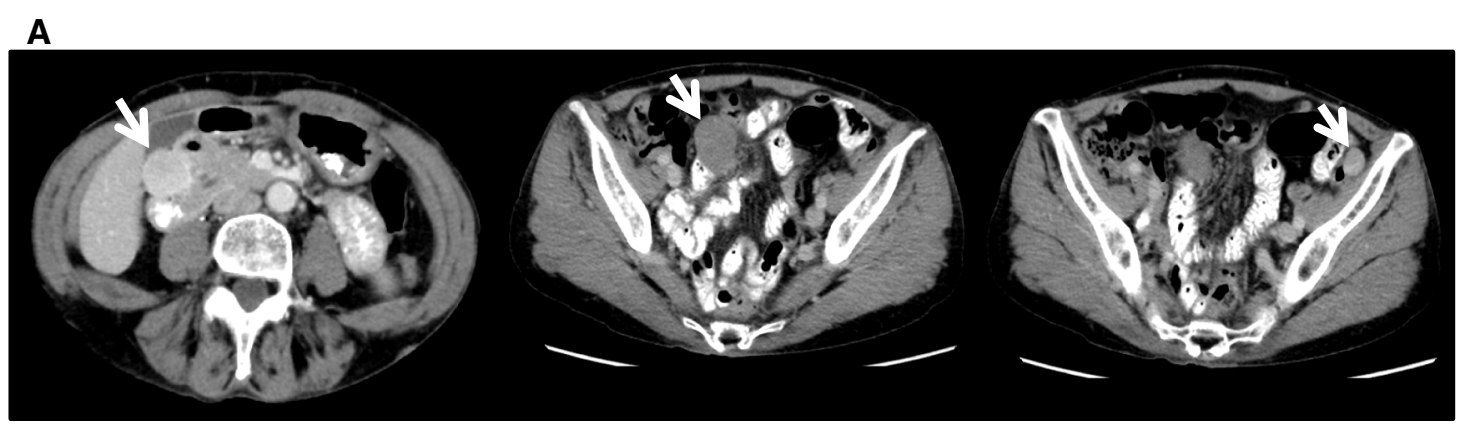

B

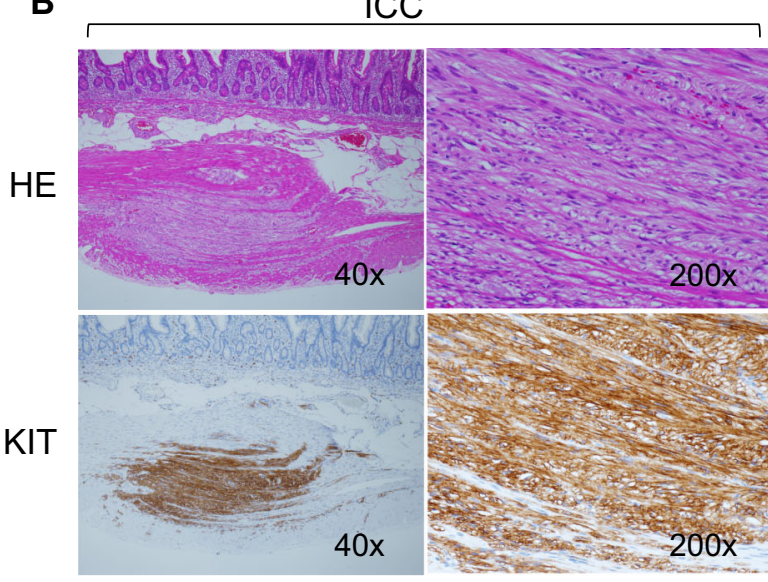

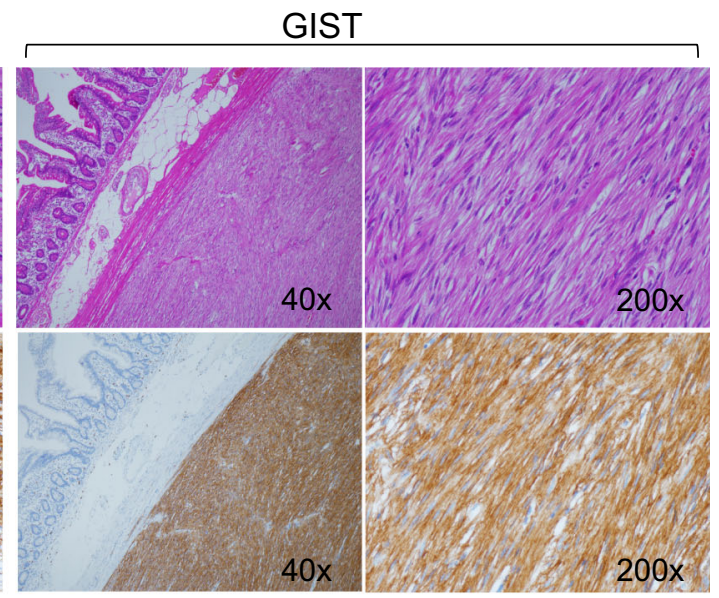

GIST (right) observed in an NF1 patient with GISTs. The upper figures show H\&E staining, and the lower figures show KIT immunostaining. " $\times 40$ " and " $\times 200$ " indicate the magnification of the objective lens tive case of an NF-1 patient whose multiple GISTs were detected by MDCT screening. The patient was a 64-year-old female without signs or symptoms. The arrows indicate multiple intestinal GISTs. b Demonstrates ICC hyperplasia (left) in the normal jejunum and a

Table 2 Characteristic features of NF1-GISTs found by the MDCT screening

\begin{tabular}{|c|c|c|c|c|c|c|c|c|c|}
\hline \multirow[t]{2}{*}{ Age } & \multirow[t]{2}{*}{ Sex } & \multirow[t]{2}{*}{ Organs } & \multirow[t]{2}{*}{ Multiplicity } & \multirow[t]{2}{*}{ Max size $(\mathrm{cm})$} & \multirow[t]{2}{*}{ ICC hyperplasia } & \multirow[t]{2}{*}{ Cell type } & \multirow[t]{2}{*}{ Mitosis (/50HPF) } & \multicolumn{2}{|c|}{ Mutations } \\
\hline & & & & & & & & $K I T$ & PDGFRA \\
\hline 66 & M & $\mathrm{D}, \mathrm{J}$ & Yes & 4 & Yes & Spindle & 0 & None & None \\
\hline 60 & M & $\mathrm{J}$ & No & 6 & Yes & Spindle & 1 & None & None \\
\hline 38 & $\mathrm{~F}$ & $\mathrm{~J}$ & Yes & 3 & Yes & Spindle & 0 & None & None \\
\hline 51 & M & $\mathrm{D}, \mathrm{J}$ & Yes & 1.8 & Yes & Spindle & 1 & None & None \\
\hline 31 & M & $\mathrm{J}, \mathrm{I}$ & Yes & 3 & Yes & Spindle & 0 & None & None \\
\hline 64 & $\mathrm{~F}$ & $\mathrm{D}, \mathrm{J}$ & Yes & 4.2 & Yes & Spindle & 0 & None & None \\
\hline
\end{tabular}

$D$ duodenum, $J$ jejunum, $I$ ileum

was significantly lower for mitotic counts (/50 HPF) and Ki67-positive tumor cells than that of the non-NF1-GISTs. KIT or PDGFRA mutations were not observed in the studied NF1-GISTs while 294 of the 303 non-NF1-GISTs (97.0\%) exhibited KIT or PDGFRA mutations. In pathological examinations, NF1-GIST cells appeared more spindle-shaped, but this finding was not statistically significant. Thus, no significant differences were observed between the 2 populations with respect to their age, gender, tumor size, recurrence, cell type, or KIT-immunoreactivity.
With a median follow-up of 3.6 years, 4 NF1-GIST patients $(18.2 \%)$ had relapses in their GISTs, and 225 nonNF1 $(24.9 \%)$ patients had recurrences (Table 3). Peritoneal recurrence was the primary relapse site for all 4 NF1-GIST patients. Among the 7 NF1-GIST patients who underwent R2 surgery, only 2 patients had recurrences during a median follow-up of 4.3 years. No significant difference was observed in recurrence risk between the two groups $(p=0.5946)$. Two of the 4 recurrent NF1-GIST patients received imatinib (400 $\mathrm{mg} / \mathrm{day})$, and both showed 
Table 3 Clinicopathologic features of NF-1 GISTs and sporadic GISTs

\begin{tabular}{|c|c|c|c|c|}
\hline & \multicolumn{2}{|l|}{ NF-1 GIST } & \multirow{2}{*}{$\begin{array}{l}\text { Sporadic GIST } \\
\text { Japanese set }\end{array}$} & \multirow[t]{2}{*}{$p$ values ${ }^{\mathrm{a}}$} \\
\hline & Japanese set & French set & & \\
\hline No. of NF1-GISTs & 22 & 18 & 904 & \\
\hline Median follow-up (yrs) & 3.3 & & 3.6 & \\
\hline Median age at $\mathrm{d} x$ & $61(26-77)$ & $51(30-67)$ & $64(10-93)$ & 0.4302 \\
\hline \multicolumn{5}{|l|}{ Gender } \\
\hline Male & 9 & 6 & 481 & \multirow[t]{2}{*}{0.3543} \\
\hline Female & 13 & 12 & 423 & \\
\hline Median size $(\mathrm{cm})$ & $4.1(1.8-21)$ & $6.6(1.7-31)$ & $5.0(0.7-35)$ & 0.5519 \\
\hline \multicolumn{5}{|l|}{ Multiplicity } \\
\hline No & 6 & 0 & 867 & \multirow[t]{3}{*}{$<0.0001$} \\
\hline Yes & 16 & 0 & 17 & \\
\hline Not available & 0 & 18 & 20 & \\
\hline \multicolumn{5}{|l|}{ Location } \\
\hline Esophagus & 0 & 0 & 9 & \multirow[t]{5}{*}{$<0.0001$} \\
\hline Stomach & 0 & 1 & 623 & \\
\hline Intestine & 22 & 15 & 213 & \\
\hline Colon & 0 & 0 & 52 & \\
\hline Others & 0 & 2 & 7 & \\
\hline \multicolumn{5}{|l|}{ Curability $^{\mathrm{b}}$} \\
\hline $\mathrm{R} 0, \mathrm{R} 1$ & 15 & 7 & 784 & \multirow[t]{3}{*}{$0.0119^{\mathrm{d}}$} \\
\hline $\mathrm{R} 2$ & 7 & 1 & 104 & \\
\hline Not available & 0 & 10 & 16 & \\
\hline \multicolumn{5}{|l|}{ Mutations $^{\mathrm{c}}$} \\
\hline Yes & 0 & 0 & 294 & \multirow[t]{3}{*}{$<0.0001$} \\
\hline No & 8 & 6 & 9 & \\
\hline Not available & 14 & 12 & 601 & \\
\hline \multicolumn{5}{|l|}{ Recurrence } \\
\hline No & 18 & 8 & 679 & \multirow[t]{3}{*}{$0.6382^{\mathrm{d}}$} \\
\hline Yes & 4 & 1 & 225 & \\
\hline Not available & 0 & 9 & 0 & \\
\hline \multicolumn{5}{|l|}{ Prognosis (OS) } \\
\hline Alive & 20 & 11 & 734 & \multirow[t]{3}{*}{$0.3790^{\mathrm{d}}$} \\
\hline Dead & 2 & 0 & 170 & \\
\hline Not available & 0 & 7 & 0 & \\
\hline \multirow[t]{2}{*}{ Mitosis (/50HPF) } & $0.0(0-8)$ & $1(0-3)$ & $3.0(0-300)$ & \multirow[t]{2}{*}{0.0093} \\
\hline & $(n=22)$ & $(n=3)$ & $(n=902)$ & \\
\hline \multirow[t]{2}{*}{ Ki67 (\%) } & $0.5(0.5-15)$ & NA & $2.5(0-50)$ & \multirow[t]{2}{*}{0.0022} \\
\hline & $(n=21)$ & & $(n=248)$ & \\
\hline \multicolumn{5}{|l|}{ Cell type } \\
\hline Spindle & 21 & 4 & 671 & \multirow[t]{3}{*}{0.0636} \\
\hline Epithelioid or mixed & 1 & 0 & 75 & \\
\hline Not available & 0 & 14 & 158 & \\
\hline
\end{tabular}

NA not available

a Comparison between Japanese NF-1 GISTs and Japanese sporadic GISTs

b Curability of surgery

${ }^{c}$ Mutations in the KIT and PDGFRA genes

d By the Fisher's exact test 

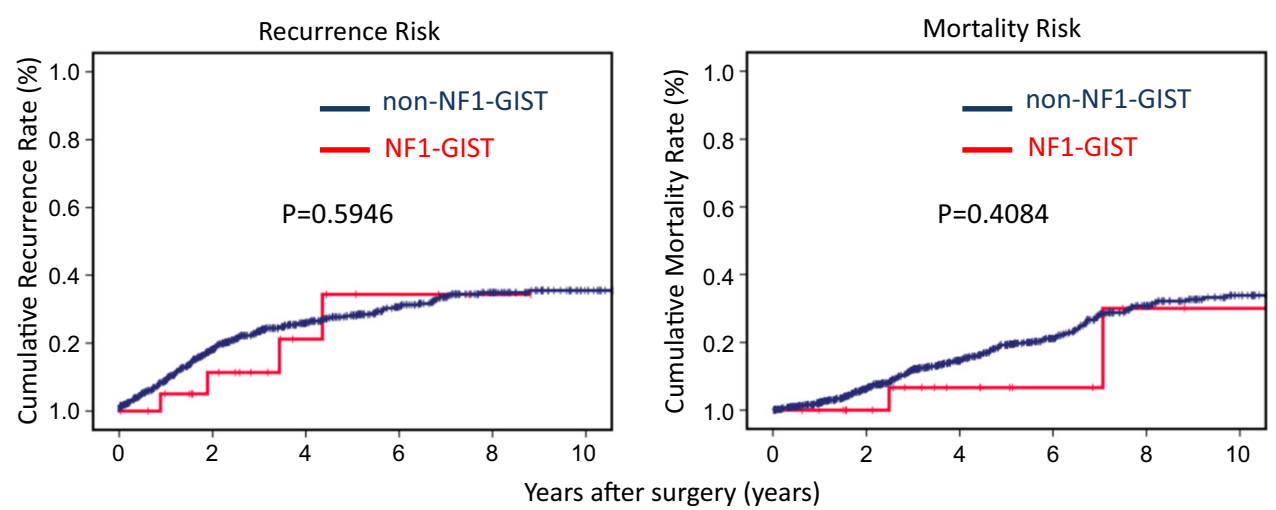

\begin{tabular}{lrrr}
\multicolumn{4}{l}{ The number of patients at risk } \\
Year & 0 & 2 & 4 \\
NF1 & 22 & 14 & 6 \\
non-NF1 & 903 & 548 & 336
\end{tabular}

Fig. 2 NF1-GIST patient prognosis. The cumulative recurrence rate (right panel) and cumulative mortality rate (left panel) are shown. The median RFS was not reached in the NF1-GIST group, but the

$\begin{array}{lrrrrrr}\text { Year } & 0 & 2 & 4 & 6 & 8 & 10 \\ \text { NF1 } & 22 & 16 & 9 & 5 & 2 & 1 \\ \text { non-NF1 } & 903 & 651 & 418 & 261 & 159 & 102\end{array}$

median RFS in the non-NF1-GIST group was estimated at 19.0 years. Median OS was not reached in both groups

clinical incidence of GISTs, and particularly intestinal GISTs, may vary, as suggested by the number of asymptomatic GISTs identified and studied here. Indeed, NF1GIST incidence may have been estimated to be higher in a report where half of the NF1-GISTs were incidentally discovered [14], whereas another report estimating a lower incidence involved predominantly NF1-GIST patients with gastrointestinal symptoms [8]. Furthermore, all these reports retrospectively collected NF1-GISTs. In the present study, we prospectively examined the intra-abdominal tumors of NF-1 patients by MDCT and observed that 6 of 95 NF1 patients exhibited small-intestinal GISTs. Furthermore, 5 of 6 patients were asymptomatic, suggesting that a significant number of NF1 patients in middle age could potentially have subclinical intestinal GISTs. The prevalence rate is $6.3 / 100 \mathrm{NF} 1$ patients and is similar to that obtained by the Swedish Cancer Registry [11]. In our study, the incidence rate was estimated at $6.3 / 1000 / \mathrm{NF} 1$ patients/year, and the target NF1 patients were typically between 30 and 60 years old. Thus, GIST incidence in the NF1 patients was estimated at 200-fold higher than that for sporadic GISTs, which was postulated at 15 cases per year per million individuals. Although the usefulness of MDCT screening for intestinal tumors has not been established, MDCT can detect intestinal masses larger than $2 \mathrm{~cm}$ $[16,21]$.

From our study, NF1-GISTs accounted for 1-2\% of all GISTs, which is consistent with previous reports [8, 14]. Thus, the incidence rate and the proportion of NF1-GISTs in sporadic GISTs appear very similar in Japanese and Caucasian populations. Most NF1-GISTs are located in the small intestine, including the duodenum and proximal jejunum, and frequently present as multiple tumors with an 
indolent nature. Contrary to our observations, some studies indicated that the age of GIST diagnosis tends to be younger for NF1 patients than for patients with sporadic GISTs [8, 14]. The mitotic counts for NF1-GISTs were lower than those for sporadic GISTs in this study; however, the observed tumor size was similar, contradicting the results of a previous study without a control arm [8]. Many reports, including this study, have observed the majority of NF1-GISTs lack KIT and PDGFRA mutations, [3, 6-10, $12-15,22]$ and accordingly, NF1-GISTs might not respond to imatinib [10].

Neurofibromin is a negative regulator of Ras kinases, and its loss of function may activate Ras and downstream kinases, including those in the MEK-MAPK pathway [3]. The MEK-MAPK pathway and the subsequent expression of ETV1, a master regulator of an ICC-GIST-specific transcription network and KIT expression [23], is predominantly activated in NF1-GISTs [15], which may result in strong KIT-positivity in immunohistochemistry. In this study, NF1 patients with GISTs exhibited ICC hyperplasia in the myenteric plexus of the small intestine, as observed in familial GIST patients with germline KIT mutations [24]. More than half of NF1-GIST patients exhibited multiple GISTs in the small intestine [8, 10]. These observations may be related to the high incidence of GISTs in NF1 patients. A previous report investigating clonality in familial GIST patients with germline KIT mutations indicated that cells in the ICC hyperplasia have polyclonal proliferation, whereas GIST tumor cells show a monoclonal development [25].

This study indicated that NF1-GIST patients underwent more frequent R2 surgery than non-NF1-GIST patients and that NF1-GIST relapses were mainly peritoneal disease (Table 3). These results suggest that multiple occurrences of intestinal GISTs may be responsible for incomplete surgery and peritoneal recurrence in NF1 patients. However, the recurrence rate in NF1 patients was similar to that of nonNF1-GIST patients (Fig. 2). The OS of NF1-GIST patients was not inferior to that of sporadic GIST patients, although imatinib failed to show any activity on recurrent NF1-GISTs [10]. Furthermore, when the prognosis was compared with the condition of small-intestinal GISTs, the RFS and OS of the NF1-GIST patients appeared superior to those of the nonNF1-GIST patients (Supplemental Fig. 1). These results could be from the indolent nature and low proliferation activity of the NF1-GISTs [7, 10]. In fact, the mitotic count and Ki67 staining of the NF1-GISTs were significantly lower than those of the other sporadic GISTs, and 5 NF1-GIST patients with R2 surgery had no evidence of recurrence at the time of analysis, with a median follow-up of 2.5 years.

In summary, we have prospectively evaluated the incidence of GISTs in NF-1 patients. The prevalence rate of GISTs was estimated at nearly $6 \%$ in adult NF1 patients, and NF1-GISTs may account for 1-2 \% of total sporadic GISTs. The risk of developing GISTs in NF1 patients is estimated at nearly 200-fold higher than that in normal populations. The clinical, pathologic and genetic features of NF1-GISTs differ from those of sporadic GISTs, including the development of multiple small intestinal tumors, an absence of KIT and PDGFRA mutations, and an indolent nature. Although the NF1-GISTs are frequently accompanied with multiple tumors and R2 surgery, the RFS and OS of NF1-GIST patients are similar to those of the normal population with sporadic GISTs.

Acknowledgments We would like to thank the staff at each hospital and institution for collecting data for this study. Data collection collaborators included Drs. Takeshi Omori, Osaka Police Hospital; Takashi Ishikawa, Niigata University Medical and Dental Hospital; Masahiro Yamamura, Kawasaki Medical School; Shinya Tokunaga, Osaka City General Hospital; Syunji Endo, Higashiosaka Municipal Hospital; Hirofumi Noro, Otemae Hospital; Hiroshi Imamura, Toyonaka Municipal Hospital; Atsushi Takeno, Kansai Rosai Hospital; Kazuo Kinoshita, Sumitomo Hospital; and Masashi Yamamoto, Kobe University Hospital; and Jean-Francois Emile, Université de Versailles Saint-Quentin. The work is supported by the Japanese Study Group on GIST and The Research Group for Rare Neoplasms of Japan. This work is supported in part by an Grant-in-Aid (H26Kakushingann-Ipann-089) from the Ministry of Health, Labor, and Welfare, Grants-in-Aid (26640110) for Scientific Research from the Ministry of Education, Culture, Sports, Science and Technology, Japan, and by the National Cancer Centre Research and Development Fund (26-A-21). For NetSARC, it is supported in part by Grants from the French INCa: NETSARC, RREPS, LYRIC-INCA-DGOS-4664, as well as from the FP7 Eurosarc (FP7-278472).

\section{Compliance with ethical standards}

Conflict of interest T. Nishida has received funding for basic research from Novartis and Bayer. JY Blay received research support and honoraria from Novartis, Pfizer and Bayer. The others have no COI.

Open Access This article is distributed under the terms of the Creative Commons Attribution 4.0 International License (http://crea tivecommons.org/licenses/by/4.0/), which permits unrestricted use, distribution, and reproduction in any medium, provided you give appropriate credit to the original author(s) and the source, provide a link to the Creative Commons license, and indicate if changes were made.

\section{References}

1. Corless CL, Barnett CM, Heinrich MC. Gastrointestinal stromal tumours: origin and molecular oncology. Nat Rev Cancer. 2011;11:865-78.

2. Joensuu H, Hohenberger P, Corless CL. Gastrointestinal stromal tumour. Lancet. 2013;382:973-8.

3. Brems H, Beert E, de Ravel T, Legius E. Mechanisms in the pathogenesis of malignant tumours in neurofibromatosis type 1 . Lancet Oncol. 2009;10:508-15.

4. Ferner RE, Huson SM, Thomas N, et al. Guidelines for the diagnosis and management of individuals with neurofibromatosis 1. J Med Genet. 2007;44:81-8. 
5. Brasfield RD. Das Gupta TK. von Recklinghausen's disease: a clinicopathological study. Ann Surg. 1972;175:86-104.

6. Cheng SP, Huang MJ, Yang TL, et al. Neurofibromatosis with gastrointestinal stromal tumors: insights into the association. Dig Dis Sci. 2004;49:1165-9.

7. Takazawa Y, Sakurai S, Sakuma Y, et al. Gastrointestinal stromal tumors of neurofibromatosis type I (von Recklinghausen's disease). Am J Surg Pathol. 2005;29:755-63.

8. Miettinen M, Fetsch JF, Sobin LH, et al. Gastrointestinal stromal tumors in patients with neurofibromatosis 1: a clinicopathologic and molecular genetic study of 45 cases. Am J Surg Pathol. 2006;30:90-6.

9. Lee JL, Kim JY, Ryu MH, et al. Response to imatinib in KIT- and PDGFRA-wild type gastrointestinal stromal associated with neurofibromatosis type 1. Dig Dis Sci. 2006;51:1043-6.

10. Mussi C, Schildhaus HU, Gronchi A, et al. Therapeutic consequences from molecular biology for gastrointestinal stromal tumor patients affected by neurofibromatosis type 1 . Clin Cancer Res. 2008;14:4550-5.

11. Zöller ME, Rembeck B, Odén A, et al. Malignant and benign tumors in patients with neurofibromatosis type 1 in a defined Swedish population. Cancer. 1997;79:2125-31.

12. Kinoshita K, Hirota S, Isozaki K, et al. Absence of c-kit gene mutations in gastrointestinal stromal tumours from neurofibromatosis type 1 patients. J Pathol. 2004;202:80-5.

13. Yantiss RK, Rosenberg AE, Sarran L, et al. Multiple gastrointestinal stromal tumors in type I neurofibromatosis: a pathologic and molecular study. Mod Pathol. 2005;18:475-84.

14. Andersson J, Sihto H, Meis-Kindblom JM, et al. NF1-associated gastrointestinal stromal tumors have unique clinical, phenotypic, and genotypic characteristics. Am J Surg Pathol. 2005;29:1170-6.

15. Maertens O, Prenen H, Debiec-Rychter M, et al. Molecular pathogenesis of multiple gastrointestinal stromal tumors in NF1 patients. Hum Mol Genet. 2006;15:1015-23.
16. Nishida T, Kumano S, Sugiura T, et al. Multidetector CT of highrisk patients with occult gastrointestinal stromal tumors. Am J Roentogenol. 2003;180:185-9.

17. Hirota S, Isozaki K, Moriyama Y, et al. Gain-of-function mutations of c-kit in human gastrointestinal stromal tumors. Science. 1998;279:577-80.

18. Rubin BP, Singer S, Tsao C, et al. KIT activation is a ubiquitous feature of gastrointestinal stromal tumors. Cancer Res. 2001;61:8118-21.

19. Hirota S, Ohashi A, Nishida T, et al. Gain-of-function mutations of platelet-derived growth factor receptor alpha gene in gastrointestinal stromal tumors. Gastroenterology. 2003;125:660-7.

20. Ghrist TD. Gastrointestinal involvement in neurofibromatosis. Arch Intern Med. 1963;112:357-62.

21. Jang KM, Min K, Kim MJ, et al. Diagnostic performance of CT in the detection of intestinal ischemia associated with smallbowel obstruction using maximal attenuation of region of interest. AJR Am J Roentgenol. 2010;194:957-63.

22. Yamamoto H, Tobo T, Nakamori M, et al. Neurofibromatosis type 1-related gastrointestinal stromal tumors: a special reference to loss of heterozygosity at $14 \mathrm{q}$ and $22 \mathrm{q}$. J Cancer Res Clin Oncol. 2009;135:791-8.

23. Chi P, Chen Y, Zhang L, et al. ETV1 is a lineage survival factor that cooperates with KIT in gastrointestinal stromal tumours. Nature. 2010;467:849-53.

24. Nishida T, Hirota S, Taniguchi M, et al. Familial gastrointestinal stromal tumours with germline mutation of the KIT gene. Nat Genet. 1998;19:323-4.

25. Chen H, Hirota S, Isozaki K, et al. Polyclonal nature of diffuse proliferation of interstitial cells of Cajal in patients with familial and multiple gastrointestinal stromal tumours. Gut. 2002;51:793-6. 